\title{
A Polyamine-sensitive Mutant of Aspergillus nidulans
}

\author{
By D. H. SPATHAS, $\dagger$ A. J. CLUTTERBUCK* and J. A. PATEMAN \\ Department of Genetics, University of Glasgow, Glasgow G11 5JS, U.K.
}

(Received 1 November 1982)

\begin{abstract}
A mutation designated sps $A 1$ has been induced in the putrescine ( $p u A 2$ ) auxotroph of Aspergillus nidulans which enables this mutant to grow on low concentrations of spermidine in place of putrescine. In addition, the sps $A I$ mutant, irrespective of putrescine requirement, is abnormally sensitive to high concentrations of spermidine, spermine or the polyamine analogue methylglyoxal bis(guanylhydrazone). When $\operatorname{sps} A I$ strains are grown on medium containing spermidine, uptake of the polyamine continues at a high level for a longer period than in the wild-type and leads to a doubled intracellular spermidine pool. A similar increase in the intracellular spermine pool results from growth on spermine.
\end{abstract}

\section{INTRODUCTION}

Mutants at the $p u A$ locus of Aspergillus nidulans are unable to synthesize polyamines because they are deficient in ornithine decarboxylase (Stevens, 1975). Both Sneath (1955) and Hope \& Stevens (1976) found that growth of the puAl mutant is supported by $0.06 \mathrm{~mm}$-putrescine but although the only known function of putrescine is to serve as a precursor of spermidine, spermidine will only support growth of $p u A$ mutants at a ten times higher concentration. Spermine is required at even higher concentrations to have any effect at all. These differences would suggest either permeability barriers for spermidine, as has been found in Neurospora crassa (Paulus \& Davis, 1981) or an additional function for putrescine other than its conversion to the higher polyamines.

We have shown that spermidine is taken up less effectively than putrescine (Spathas et al., 1982) but in order to investigate this situation further, we have isolated secondary mutants in the $p u A 2$ auxotroph which enable it to grow on low concentrations of spermidine comparable to those required for putrescine. The properties of these mutants are reported here.

\section{METHODS}

Materials. Hydrochloride salts of putrescine, spermidine and spermine were obtained from Sigma. Methylglyoxal bis(guanylhydrazone) dihydrochloride monohydrate (MGBG), a potent inhibitor of $S$-adenosyl-Lmethionine decarboxylase (Williams-Ashman \& Schenone, 1972) was obtained from Aldrich Chemical Co. ${ }^{14} \mathrm{C}$ Labelled polyamines were obtained from Amersham. Dansyl chloride was a BDH preparation.

Media. Minimal medium and supplements for standard mutants were those described by Pontecorvo et al. (1953), sodium nitrate being altered in concentration or replaced with other nitrogen sources where stated. DifcoBacto agar was used to solidify media for growth tests. Liquid minimal medium was as described by Cove (1966).

Strains and genetic analysis. Techniques were those described by Pontecorvo et al. (1953) and McCully \& Forbes (1965). The biotin requiring strain biAl (Glasgow no. G051) was regarded as the wild-type for the purpose of these studies. Glasgow strain $\mathrm{G} 0171$ is a putrescine auxotroph biAl;puA2 isolated by $\mathrm{C}$. Herman (personal communication); $p u A 2$ is allelic to the $p u A I$ mutant isolated by Sneath (1955). For uptake assays and polyamine pool estimations, the wild-type strains $b i A I$ or biAl;wA3 were compared with the recombinant strains of genotype biAl;wA3;spsAI and pabaAI;yA2;spsAI which were obtained by outcrossing the biAI;puA2;spsAI mutant (see Results). Master strain E (Glasgow no. G95) was used for mitotic mapping (McCully \& Forbes, 1965).

† Present address: Department of Biology, University of Patras Medical School, Patras, Greece.

$\ddagger$ Present address: Department of Genetics, Research School of Biological Sciences, Australian National University, Box 475, Canberra, A.C.T. 2601, Australia. 
Mutant isolation. A dense conidial suspension of the biAl;puA2 strain was treated with nitrous acid (Siddiqi, 1962 ) to give a $45 \%$ survival rate. The mutagenized conidia were plated at suitable dilutions on minimal medium supplemented with biotin and 0.06 mM-spermidine; the colonies which grew after $2-3 \mathrm{~d}$ incubation at $37^{\circ} \mathrm{C}$ were isolated, purified and classified by stab inoculation on to appropriate media.

Polyamine uptake assays. The $5 \mathrm{~min}$ uptake assays from $3 \mathrm{~mm}{ }^{14} \mathrm{C}$-labelled polyamine solutions were carried out as described by Spathas et al. (1982). For the longer term uptake experiment (Fig. 2) the following modifications were made to the procedure. The mycelium was grown for $17 \mathrm{~h}$ as previously and was then decanted into fresh medium containing $10 \mathrm{mM}$-nitrate for a further hour, and the uptake assays were also made in this medium. For this purpose the culture was divided into $5 \mathrm{ml}$ samples containing approximately $80 \mathrm{mg}$ wet weight of mycelium, and shaken in $25 \mathrm{ml}$ flasks in a $30^{\circ} \mathrm{C}$ water bath. After addition of $\left[{ }^{14} \mathrm{C}\right]$ spermidine and incubation for the appropriate time, the mycelium was filtered and washed as before, but was then air-dried before weighing. The radioactivity was counted in Biofluor scintillation fluid (New England Nuclear).

Cell polyamine pools. Mycelium was grown, as for the uptake assays, in $10 \mathrm{~mm}$-nitrate medium shaking cultures for $17 \mathrm{~h}$ at $30^{\circ} \mathrm{C}$ and was then shaken for a further $3 \mathrm{~h}$ in the specified treatment medium (Table 3). The mycelium was filtered off, washed and freeze-dried. The polyamines in the freeze-dried material were then extracted, dansylated and separated on silica gel thin layer chromatograms by the method of Dion \& Herbst (1970). Quantitative assays were obtained by scraping off individual fluorescent spots on the chromatograms, eluting the silica gel with ethylacetate/cyclohexane $(2: 3, \mathrm{v} / \mathrm{v})$ and assaying the fluorescence in comparison with dansylated polyamine standards using a Fluoripoint fluorimeter.

\section{RESULTS}

\section{Isolation and properties of mutants}

We have isolated by mutagenesis of the biA1;puA2 putrescine auxotroph a number of strains which were able to grow on low concentrations of spermidine in place of putrescine. Only one of these has been studied in detail: its phenotype was found to be due to a mutation, designated spaAl which was also shown to confer sensitivity to higher concentrations of spermidine (Table 1 and Fig. 1). As a result of this mutation the biAl;puA2;spsA1 strain can grow on $0.06 \mathrm{~mm}$ spermidine, but $10 \mathrm{mM}$-spermidine, which is the concentration required to support the growth of the biA1;puA2 parent strain, is inhibitory to the new mutant, as it is to a biA1;spsA1 recombinant obtained by outcrossing to remove the putrescine requirement. In order to check that the two properties of spsA1 strains were due to a single mutation, 20 spermidine-sensitive, putrescine-requiring segregants were picked from crosses in which biAl;puA2;spsAl was one of the parents: all retained the ability to grow on $0.06 \mathrm{mM}$-spermidine in place of putrescine.

Figure 1 also illustrates the effect of spermine on these strains. Spermine alone did not effectively support the growth of either $p u A 2$ or $p u A 2 ; s p s A 1$ strains, but it did have a sparing effect on the utilization of putrescine or spermidine, and this effect is enhanced in the spsAI mutant. Spermine, like spermidine is also toxic to spsAl strains at high concentration.

Another class of mutants, one example of which is shown under the designation spd-6 in Table 1 , also gave some growth on low spermidine concentrations, but was not inhibited by high spermidine. This could be due to a less extreme allele of $s p s A l$ or a mutant at a different locus.

The response of sps $A I$ strains to both high and low polyamine concentrations shown in Table 1 was unaltered if the nitrogen source ( $10 \mathrm{~mm}$-nitrate) was replaced by $10 \mathrm{~mm}$ concentrations of nitrite, alanine, glutamate, asparagine, proline, arginine or by nitrate up to $70 \mathrm{mM}$ or urea up to $100 \mathrm{~mm}$. However, $10 \mathrm{~mm}$-ammonium prevented the growth of puA2;spsAI and puA2 spd-6 strains on low spermidine concentrations (Table 2) and $10 \mathrm{mM}$-methylammonium or $70 \mathrm{~mm}$ nitrate plus $1 \mathrm{~mm}$-ammonium had a similar effect. Higher ammonium concentrations (or nitrate plus ammonium combinations) were required to reverse the toxicity of spermidine to sps $A 1$. High salt ( $1 \mathrm{M}-\mathrm{KCl}$ and $0.7 \mathrm{M}-\mathrm{NaH}_{2} \mathrm{PO}_{4}$ were tested) had similar effects. None of these medium modifications affected the response of the puA2 strain to spermidine, nor did they prevent the growth of $p u A 2$, or the mutants derived from it on putrescine.

Putrescine is a moderately effective nitrogen source for $A$. nidulans while spermidine and spermine are not (Spathas et al., 1983). This situation is unchanged in spsAl strains, and the utilization of putrescine as nitrogen source is unaffected by salt concentration.

The putrescine analogue MGBG has two effects: at $1 \mathrm{~mm}$ concentrations, it prevents the 
Table 1. Growth responses of spermidine utilizing derivatives of the putrescine auxotroph (puA2) of A. nidulans

Supplements were added to standard minimal medium plus biotin.

\begin{tabular}{|c|c|c|c|c|c|}
\hline \multirow[b]{2}{*}{ Supplement } & \multicolumn{5}{|c|}{ 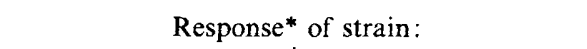 } \\
\hline & $\begin{array}{c}\text { biAl } \\
\text { (wild- } \\
\text { type) }\end{array}$ & $\begin{array}{l}\text { biAl } \\
\text { puA2 }\end{array}$ & $\begin{array}{l}\text { biAl } \\
\text { puA2 } \\
\text { spsAI }\end{array}$ & $\begin{array}{l}\text { biAl } \\
\operatorname{sps} A 1\end{array}$ & $\begin{array}{c}b i A 1 \\
\text { puA2 } \\
\text { spd-6 }\end{array}$ \\
\hline None & + & - & - & + & - \\
\hline $0.06 \mathrm{~mm}$-Putrescine & + & + & + & + & + \\
\hline $0.06 \mathrm{mM}$-Spermidine & + & - & + & + & + \\
\hline 10 mM-Spermidine & + & + & - & - & + \\
\hline
\end{tabular}

* + Denotes good growth; - denotes no growth.
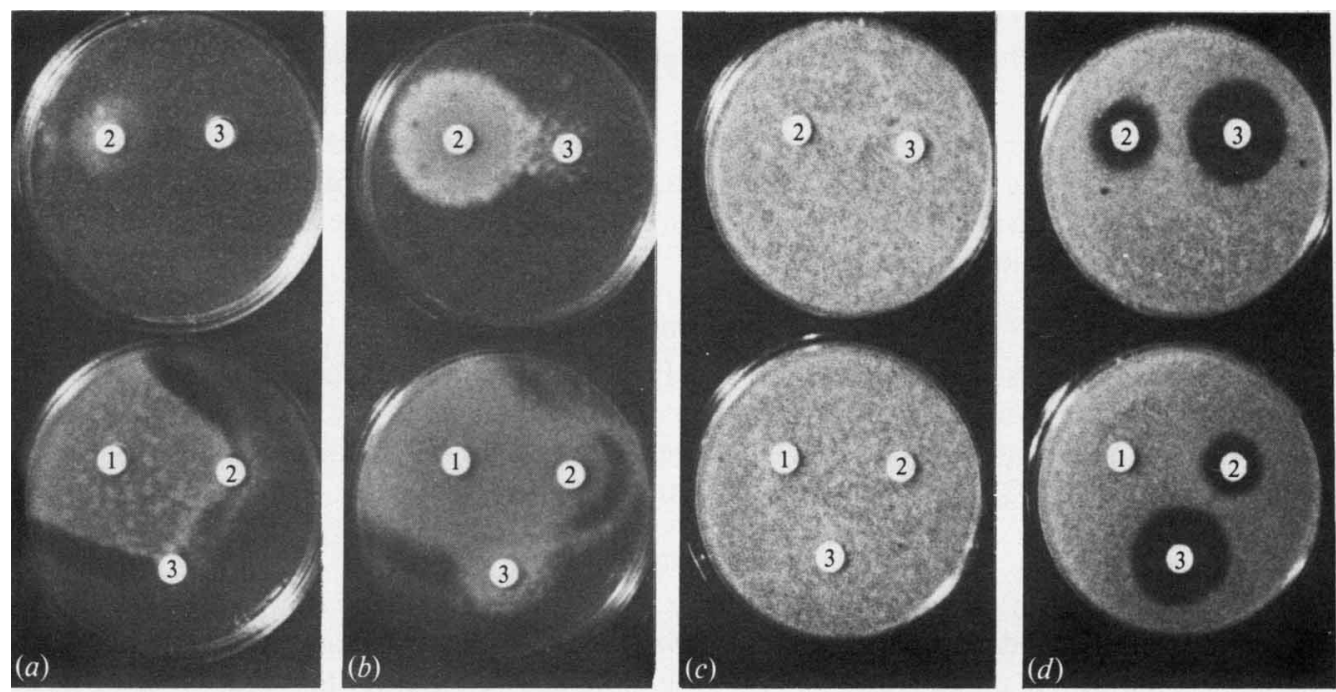

Fig. 1. Auxanographic responses of $A$. nidulans strains to polyamines ( $a$, strain $b i A I ; p u A 2 ; b$, strain biAl;puA2;spsAl;c, strain biAl;d,biAl;spsA1). Conidia of the appropriate strain were suspended in minimal agar plus biotin. The wells contained: putrescine (1), spermidine (2) or spermine (3). (a) and (b) show utilization of various polyamines by strains unable to synthesize their own; each well contained $60 \mathrm{nmol}$ of polyamine. $(c)$ and $(d)$ show inhibition of non-requiring strains by higher concentrations of polyamines: $150 \mathrm{nmol}$ putrescine (which is not inhibitory at any concentration) or $10 \mu \mathrm{mol}$ spermidine or spermine.

growth of $p u A 2$ strains on $0.06 \mathrm{~mm}$-putrescine, whether or not they carry spsAl or spd-6. This inhibition is apparently competitive since it is reversed by higher concentrations of putrescine. In addition, $10 \mathrm{mM}-\mathrm{MGBG}$ prevents the growth of all sps $A 1$ and $s p d-6$ strains whether they carry $p u A 2$ or not, and $10 \mathrm{~mm}$-putrescine does not reverse this inhibition. Thus while sps $A l$ and $s p d-6$ strains differ in that high spermidine concentrations are toxic only to the former, the mutants are similar in their sensitivity to MGBG, which means that both mutants can be classified in the absence of putrescine requirement.

A variety of comparisons were made between three biA1;wA2;spsA1 and three biA $1 ; w A 2 ; s p s A^{+}$segregants from a cross between biA1;puA2;spsA1 and a white strain (G128). The growth rate of the $s p s A 1$ segregants in shaken liquid culture was a significant $18 \%$ slower than $s p s A^{+}$segregants and no consistent variation was found within each group. These strains were also tested for sensitivity to a number of compounds diagnostic of glutathione deficiency in Escherichia coli (Murata \& Kimura, 1982) since glutathione is one of the substances which complex with polyamines (Tabor \& Tabor, 1970). The spsA1 and wild-type segregants 
Table 2. Effect of nitrogen source and salt on polyamine responses of the spsA1 and spd-6 mutants of

\section{A. nidulans}

The medium used was minimal medium in which the sodium nitrate was replaced as stated.

\begin{tabular}{|c|c|c|c|c|c|c|c|}
\hline \multirow[b]{2}{*}{$\begin{array}{l}\text { Spermidine } \\
\text { concn }\end{array}$} & \multirow[b]{2}{*}{$\begin{array}{l}\text { Nitrogen } \\
\text { source* }\end{array}$} & & \multicolumn{5}{|c|}{ Response $\dagger$ of strain: } \\
\hline & & Salt* & $\begin{array}{l}\text { biAl } \\
\text { (wild- } \\
\text { type) }\end{array}$ & $\begin{array}{l}\text { biA1; } \\
p u A 2\end{array}$ & $\begin{array}{l}\text { biAl; } \\
\text { puA2; } \\
\text { spsA1 }\end{array}$ & $\begin{array}{l}\text { biAl } \\
\operatorname{sps} A 1\end{array}$ & $\begin{array}{c}\text { biA1; } \\
\text { puA2; } \\
\text { spd-6 }\end{array}$ \\
\hline $0.06 \mathrm{mM}$ & $10 \mathrm{~mm}$-Nitrate & - & + & - & + & + & + \\
\hline $0.06 \mathrm{~mm}$ & $10 \mathrm{~mm}$-Ammonium & - & + & - & - & + & - \\
\hline $0.06 \mathrm{~mm}$ & $10 \mathrm{~mm}$-Nitrate & $1 \mathrm{M}-\mathrm{KCl}$ & + & - & - & + & - \\
\hline $6.0 \mathrm{mM}$ & $10 \mathrm{~mm}$-Nitrate & - & + & + & - & - & + \\
\hline $6.0 \mathrm{mM}$ & 70 mm-Ammonium & - & + & + & + & + & + \\
\hline $6.0 \mathrm{mM}$ & $10 \mathrm{~mm}$-Nitrate & $1 \mathrm{M}-\mathrm{KCl}$ & + & + & + & + & + \\
\hline
\end{tabular}

* See text for further details.

$\dagger+$ Denotes good growth; - denotes poor or no growth.

responded similarly to methyl glyoxal and 8-hydroxyquinoline, but mycelial growth of $\operatorname{sp} A 1$ was more retarded than the wild-type on solid media containing $8 \mu \mathrm{g}$ mercuric chloride $\mathrm{ml}^{-1}$. This result, however was easily obscured by the failure of conidia of either genotype to germinate on this concentration of inhibitor.

\section{Mapping spsAI}

In a diploid with master strain E (McCully \& Forbes, 1965), the spsAl mutant is recessive with respect to spermidine toxicity. Haploidization of this diploid showed that $\operatorname{sps} A 1$ is in linkage group III and meiotic mapping located it between galE and $c n x H$ at a distance of $23 \pm 4 \%$ from the former and $16 \pm 3 \%$ from the latter (Clutterbuck, 1982).

\section{Polyamine uptake}

Differences between $s p s A l$ and the wild-type in short term uptake $(5 \mathrm{~min})$ were investigated under a variety of conditions. Spathas et al. (1982) found that nitrogen-starved mycelium gave the highest polyamine uptake rates and investigations showed that for mycelium treated under nitrogen starvation for $3 \mathrm{~h}$, sps $A l$ had a $12 \%$ greater spermidine uptake rate than the wild-type. However, similarly increased rates were found for both spermine and putrescine although spermidine and putrescine are believed to be taken up independently (Spathas et al., 1982). Further investigations of uptake kinetics of wild-type and mutant after nitrogen starvation or growth on standard nitrate-glucose medium failed to establish significant differences. Part of this failure was due to inconsistency of results, but it could be concluded that neither the $K_{\mathrm{m}}$ nor the $V_{\max }$ for spermidine uptake differed greatly between the strains. Younger cultures $(10 \mathrm{~h}$ instead of $16 \mathrm{~h}$ ) gave similar results.

Much greater differences were obtained in a series of experiments using longer uptake times, one of which is shown in Fig. 2. This shows that while uptake rates of the two strains from $0.6 \mathrm{~mm}$-spermidine were very similar at $10 \mathrm{~min}$, the rate for sps $A$ declined much less rapidly than that of the wild-type.

\section{Cell polyamine pools}

Polyamine pools were measured in mycelium treated in various ways known to affect the growth of $s p s A l$ strains in plate tests. The results (Table 3 ) show no evidence of different pool sizes in wild-type and mutant except where polyamines, particularly spermidine or spermine were present in the medium. The clearest result comes from treatment (6) in which the mycelium was washed with $0.05 \mathrm{M}-\mathrm{HCl}$ to remove adhering polyamines. Repeated experiments consistently gave a spermidine pool in the $s p s A l$ strain double that in the wild-type. Treatments (4) and (5) confirm that spermidine and spermine pools behave similarly although the estimates include adhered polyamines. 


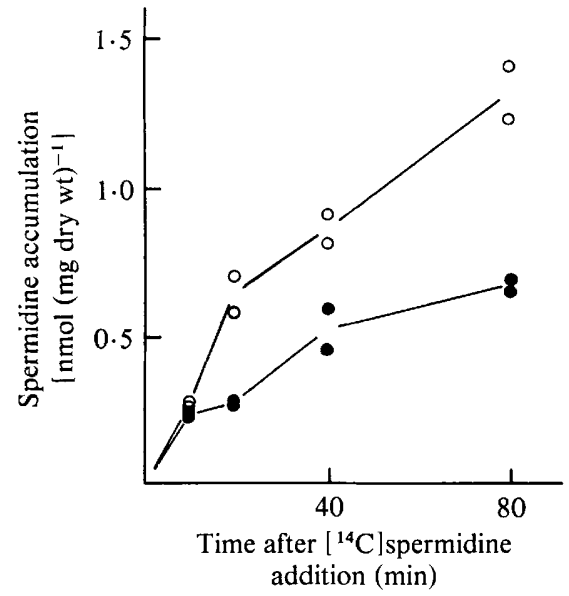

Fig. 2. Accumulation of $\left[{ }^{14} \mathrm{C}\right]$ spermidine by $\operatorname{sps} A l(O)$ and wild-type $(O)$ strains of $A$. nidulans. Mycelium was grown in minimal medium containing $10 \mathrm{~mm}$-nitrate and uptake of $\left[{ }^{14} \mathrm{C}\right]$ spermidine, provided at $0.6 \mathrm{~mm}$ concentration, was assayed in the same medium. Each point represents one assay.

\section{Table 3. Cell polyamine pools in A. nidulans wild-type and spsA1 mutant}

Mycelium was grown in $10 \mathrm{~mm}$-nitrate medium for $17 \mathrm{~h}$ and transferred to treatment medium for $3 \mathrm{~h}$ before assay of polyamines. Results for treatment 6 are the means of four estimations, and for treatment 5 , two estimations, the remainder are single estimates.

Cell polyamine pools [ $\mu \mathrm{mol}\left(\mathrm{g}\right.$ dry $\left.w \mathrm{t}^{-1}{ }^{-1}\right]$

\section{Treatment}

1. Nitrogen-free

2. $5 \mathrm{~mm}$-Nitrate

3. $5 \mathrm{~mm}$-Nitrate +5 mM-putrescine

4. $5 \mathrm{~mm}$-Nitrate +5 mM-spermidine

5. $5 \mathrm{~mm}$-Nitrate +5 mM-spermine

6. $5 \mathrm{~mm}$-Nitrate +5 mM-spermidine

\begin{tabular}{|c|c|c|c|c|c|c|}
\hline \multirow[b]{3}{*}{ Washing } & \multicolumn{6}{|c|}{ Cell polyamine pools $\left[\mu \mathrm{mol}(\mathrm{g} \text { dry } w \mathrm{t})^{-1}\right]$} \\
\hline & \multicolumn{2}{|c|}{ Putrescine } & \multicolumn{2}{|c|}{ Spermidine } & \multicolumn{2}{|c|}{ Spermine } \\
\hline & $\begin{array}{l}\text { Wild- } \\
\text { type }\end{array}$ & $\operatorname{sps} A l$ & $\begin{array}{l}\text { Wild- } \\
\text { type }\end{array}$ & $\operatorname{sps} A I$ & $\begin{array}{l}\text { Wild- } \\
\text { type }\end{array}$ & $\operatorname{sps} A I$ \\
\hline Water & $0 \cdot 7$ & $0 \cdot 6$ & $7 \cdot 0$ & $4 \cdot 5$ & $2 \cdot 0$ & $1 \cdot 2$ \\
\hline Water & 0.6 & $0 \cdot 6$ & $7 \cdot 8$ & $7 \cdot 2$ & $2 \cdot 8$ & $1 \cdot 8$ \\
\hline Water & $40 \cdot 0$ & $51 \cdot 0$ & $14 \cdot 0$ & $13 \cdot 0$ & $2 \cdot 6$ & $2 \cdot 5$ \\
\hline Water & $1 \cdot 0$ & $1 \cdot 5$ & $52 \cdot 5$ & $80 \cdot 0$ & $4 \cdot 1$ & $4 \cdot 2$ \\
\hline Water & $3 \cdot 2$ & $2 \cdot 2$ & $8 \cdot 5$ & $9 \cdot 8$ & $81 \cdot 2$ & $165 \cdot 0$ \\
\hline $0.05 \mathrm{M}-\mathrm{HCl}$ & $0 \cdot 70$ & $1 \cdot 00$ & $30 \cdot 46$ & $62 \cdot 28^{*}$ & $2 \cdot 33$ & $2 \cdot 84$ \\
\hline
\end{tabular}

*Wild-type and spsA1 are significantly different, $P<0.001$.

Comparisons of mycelial dry weight and exhaustively blotted wet weight suggest that there is $2.5 \mathrm{~g}$ of internal water per gram of dry weight, from which it can be calculated that the internal spermidine concentration may be elevated by growth on spermidine medium from $3 \mathrm{~mm}$ to $12 \mathrm{~mm}$ in the wild-type and to $25 \mathrm{~mm}$ in the spsAI strain. Even larger differences in internal spermine concentration may be inferred from treatment (5).

\section{DISCUSSION}

A striking feature of the spsAl mutant is that, in addition to its ability to use spermidine at low concentrations in place of putrescine, it is inhibited by high spermidine concentrations which are tolerated by the wild-type. It is also inhibited by spermine and the polyamine analogue MGBG. Excessive exogenous polyamines are toxic to many bacteria, possibly because of their ability to form complexes with anionic cellular components or possibly because of conversion to toxic oxidation products (see Bachrach, 1973). 
Both the ability to utilize spermidine at low concentrations and the toxicity of high concentrations are antagonized by ammonium, and to a lesser extent, nitrate ions. This suggests that spermidine uptake is inhibited and/or repressed by these nitrogen sources as it is in the wildtype (Spathas et al., 1982). The similar effects of high salt concentrations may indicate a reduction of uptake and lowering of internal pool sizes as in a number of other organisms (Munro et al., 1972, 1975).

It is still uncertain whether the uptake mechanism itself is altered in spsA1, but it is apparent from the longer term uptake experiments that the rate of spermidine accumulation declines less rapidly in the mutant than in the wild-type. There are a number of possible explanations for this. The uptake system could be relatively insensitive to feedback inhibition (or repression of synthesis) by internal polyamines, or the polyamines could fail to be converted to a form active in such feedback. On the other hand, the polyamines accumulated by spsAI could be rapidly metabolized by degradation or complex formation. This seems unlikely, however, unless the complex is very labile, since sps $A I$ was originally selected for utilization of low concentrations of spermidine in place of endogenously synthesized polyamines. Moreover, it was apparent in the $3 \mathrm{~h}$ accumulation experiments (Table 3 ) that the material taken up still behaves chromatographically as spermidine. Finally, it was suggested by Spathas et al. (1982) that exogenous polyamines may go into a separate cellular compartment which is less metabolically available than that synthesized from putrescine. If the exogenously derived compartment also regulates further uptake, all the features of spaAI could readily be explained by the hypothesis that transfer from the exogenous to the metabolic pool is accelerated in the mutant.

While it has been shown that a putrescine auxotroph can grow on low concentrations of spermidine if uptake is enhanced by the presence of a second mutation, it should be noted that this does not rule out the second possibility mentioned in the introduction - that putrescine may be required for a separate function of its own. If spermidine can be degraded, perhaps inefficiently, to putrescine, the higher intracellular concentrations of spermidine made possible by spsAI may be necessary mainly in order to force this back-reaction.

We gratefully acknowledge the excellent technical assistance of Flora Rodger.

\section{REFERENCES}

BACHRACH, U. (1973). Function of Naturally Occurring Polyamines. New York: Academic Press.

Clutterbuck, A. J. (1982). Loci and linkage map of Aspergillus nidulans. Genetic Maps 2, 208-217.

Cove, D. J. (1966). The induction and repression of nitrate reductase in the fungus Aspergillus nidulans. Biochimica et biophysica acta 113, 51-56.

Dion, A. S. \& Herbst, E. J. (1970). Polyamine changes during development of Drosophila melanogaster. Annals of the New York Academy of Sciences 171, 723-734.

Hope, J. A. \& Stevens, L. (1976). The effects of putrescine, spermidine and spermine on the growth of a polyamine-requiring mutant of Aspergillus nidulans. Biochemical Society Transactions 4, 11281130 .

KÄFER, E. (1977). Meiotic and mitotic recombination in Aspergillus and its chromosomal aberrations. Advances in Genetics 19, 33-131.

McCully, K. S. \& Forbes, E. (1965). The use of $p$ fluorophenylalanine with 'master strains' of Aspergillus nidulans for assigning genes to linkage groups. Genetical Research 6, 352-359.

Munro, G. F., Hercules, K., Morgan, J. \& SANERBIER, W. (1972). Dependence of the putrescine content of $E$. coli on the osmotic strength of the medium. Journal of Biological Chemistry 247, 12721280.

Munro, G. F., Miller, R. A., Bell, C. A. \& VERDEBER, E. L. (1975). Effect of external osmolarity on polyamine metabolism in Hela cells. Biochimica et biophysica acta 411, 263-281.

Murata, K. \& Kimura, A. (1982). Some properties of glutathione biosynthesis-deficient mutants of $E s$ cherichia coli B. Journal of General Microbiology 128, 1047-1052.

Paulus, T. J. \& Davis, R. H. (1981). Regulation of polyamine synthesis in relation to putrescine and spermidine pools in Neurospora crassa. Journal of Bacteriology 145, 14-20.

Pontecorvo, G., Roper, J. A., Hemmons, L. M, MacDonald, K. D. \& Bufton, A. W. J. (1953). The genetics of Aspergillus nidulans. Advances in Genetics 5, 141-238.

SIDDIQI, O. H. (1962). Mutagenic action of nitrous acid in Aspergillus nidulans. Genetical Research 3, 303314.

Sneath, P. H. A. (1955). Putrescine as an essential growth factor for a mutant of Aspergillus nidulans. Nature, London 175, 818. 
Spathas, D. H., Pateman, J. A. \& Clutterbuck, A. J. (1982). Polyamine transport in Aspergillus nidulans. Journal of General Microbiology 128, 557-563.

Spathas, D. H., Clutterbuck, A. J. \& Pateman, J. A. (1983). Putrescine as a nitrogen source for wild type and mutants of Aspergillus nidulans. FEMS Microbiology Letters (in the Press).

Stevens, L. (1975). Ornithine decarboxylase activity in germinating conidia of Aspergillus nidulans. FEBS Letters 59, 80-82.

TABOR, C. W. \& TABOR, H. (1966). Transport systems for 1,4-diaminobutane, spermidine and spermine in
E. coli. Journal of Biological Chemistry 241, 3714 3723.

TABOR, C. W. \& TABOR, H. (1970). The complete conversion of spermidine to a peptide derivative in E. coli. Biochemical and Biophysical Research Communications 41, 232-238.

Williams-Ashman, H. G. \& Schenone, A. (1972). Methylglyoxal bis(guanylhydrazone) as a potent inhibitor in mammalian and yeast SAM decarboxylase. Biochemical and Biophysical Research Communications 46, 288-295. 